\title{
Integrated Reasoning Engine for Code Clone Detection
}

\section{Naresh Babu Bynagari}

Andriod Developer, Keypixel Software Solutions, 777 Washington rd Parlin NJ 08859, Middlesex, USA

\begin{abstract}
This article seeks to foray into the nitty-gritty of integrated reasoning for code clone detection and how it is effectively carried out, given the amount of analytics usually associated with such activities. Detection of codes requires high-pitch familiarity with cloning systems and their workings. Hence, discovering similar code segments that are often regarded and seen as code imitations (clone) is not an easy responsibility. More especially, this very detection process might possess key purposes in the context of susceptibility findings, refactoring, and imitation detecting. Through the voyage of discovery this article intends to expose you to, you will realize that identical code segments, more often than not described as code clones, appear to be a serious duty, especially for large code bases $<1 ; 2 ; 3 ; 4>$. There are certain approaches and deep technicalities that this sort of detection is known for. Still, from the avalanche of resources that formed the bedrock of this article, one would discover the easiest formula to adopt in maneuvering such strenuous issues.
\end{abstract}

Keywords: Code Clone Lexer, Programming, Algorithms, Analytics, Duplication, Copying

\section{INTRODUCTION}

It should be borne in mind that different software engineering tasks are taking advantage of code clone detection, such as Susceptibility findings, refactoring, and imitation unraveling. Interestingly, over time, previous methodologies have been suggested to detect code clones using token subsequence compatibility, control flow enabling graph inquiry. However, despite their plausibility, it seems restricted scalability upon the pairwise joining is exorbitant in very sizable code platforms. Considering it again from another viewpoint, you will discover that experts usually propose machine learning-based clone detections to improve the former string-compatibility enabled clone findings by bringing in a new code uniformity parameter and sending the code into intermediate symbolisms (e.g., feature vectors) to uncover a lot of code imitations. Nonetheless, as it has been experimented through a series of stages, such processes can orchestrate a sizable number of false positives as a direct result of smaller code semblance, which will not ultimately produce the desirable consequence (Movva et al., 2012). 


\section{Understanding Code Clone Detection}

Code replication using the copy and paste technique without alteration into a different part of code usually happens in software development. The duplicated clone is called code clone, while the process is also referred to as code cloning. Detection of an anomaly in one section of the code, there would be a need to correct the entire duplicate segments. Thus, the identification of all the related parts throughout the source code becomes important. Also, the duplicated code increases the work to be carried during the code augmentation. According to research, between $30-50 \%$ of big software systems embody cloned codes.

\section{What is Code Clone?}

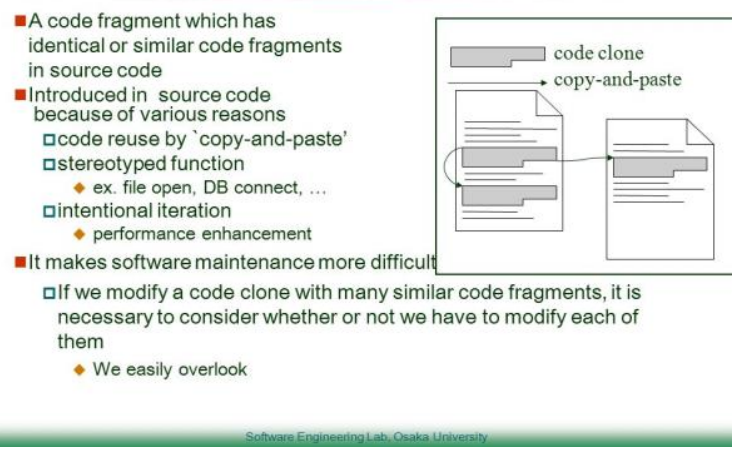

Figure 1: Code clone

Source: slideplayer.com

According to Baxter, a code clone is "a code segment that is identical to another segment." Different researchers have used different terms in referring to code cloning. Terms like duplicated code, similar codes, and so on have been used to refer to code cloning. This work provides an exhaustive review of clone detection techniques that are currently available and as tools. It also provides an in-depth study of how an integrated reasoning engine can be used for code clone detection (Narayana et al., 2012). It ought to be noticed that, as a rule, numerous computer programming assignments, for example, refactoring, appreciating code capability, or recognizing bugs, expecting the removal of linguistically comparable code pieces (normally alluded to as "code imitators"). Strangely, it appears to be that three code imitator types exist. They include:

Type 1: Indistinguishable code parts aside from varieties in identifier nomenclatures and exacting qualities;

Type 2: Linguistically comparable sections that contrast at the assertion level. The sections have proclamations added, adjusted, or eliminated regarding one another.

Type 3: Semantically unique code sections that carry out similar usefulness.

One would acknowledge like this that Code clone location approaches contain two stages in a broader premise: (I) Move code into a middle of road portrayal, for example, treebased clone recognition pronouncing highlight vectors to address code pieces; (ii) Send appropriate likeness identification calculations to identify code clones. For example, bunching calculations from AI are generally utilized in code clone recognition issues. It is likewise imperative that many existing codes clone recognition methods apply 
straightforward example coordinating (e.g., token-enabled code imitation location strategy and influence a code similitude metric to quantify the measure of closeness amongst two code tests.)

Presently, imagine that somebody needs to select code imitations for pointer-related code imitators, already operating code imitation recognition strategies seem wasteful for this reason because of the impressive measure of pointer-immaterial codes combined in connection with the objective pointers. It has likewise been seen that most progressive profound learning approaches presently neglect to separate clone tests where indicatorcentered codes are furnished in varied codecs. Consequently, there must be improved options in contrast to the present status of the workmanship arrangements. A different challenge apart from the operative imitator location strategies appears to have not ensured minute bogus positives. Nonetheless, in totally remove any hint of bogus positives, it generally requires a gigantic venture of human endeavors for additional confirmation. This will empower the legitimate investigation of the genuine positives, and the bogus positives recognized utilizing ordinary tree-based code clone location approach with various code closeness edges. One can undoubtedly see that loosening up code likeness limit can profit location with more code clone tests. In any case, the proportion of bogus positives likewise increments at that point. In case it is feasible to take out the bogus positives, whatever number could be allowed, one actually can empower a superior investigation with more clone tests.

\section{What False Positives are about?}

False Positives can be depicted in the way that when a code imitator pair is distinguished as code imitator by some sort of device meant for such identity separation, often referred to as code separator. However, two clone tests possess diverse wellbeing requirements as far as pointer investigation. For example, traditional clone recognitions, consolidating treebased methodology with AI strategies, present a code similitude estimation S, and move the code into the middle of the road portrayals to distinguish multiple code imitators. This can assist with recognizing imitators which are not indistinguishable but rather as yet possessing a comparable code architecture. Think about this genuine positive model accordingly: in tree-based imitator discovery, double source records are foremostly split and changed over into Abstract Syntax Trees (ASTs), a database harboring most of the indicator nomenclatures, and strict qualities are supplanted by AST hubs.

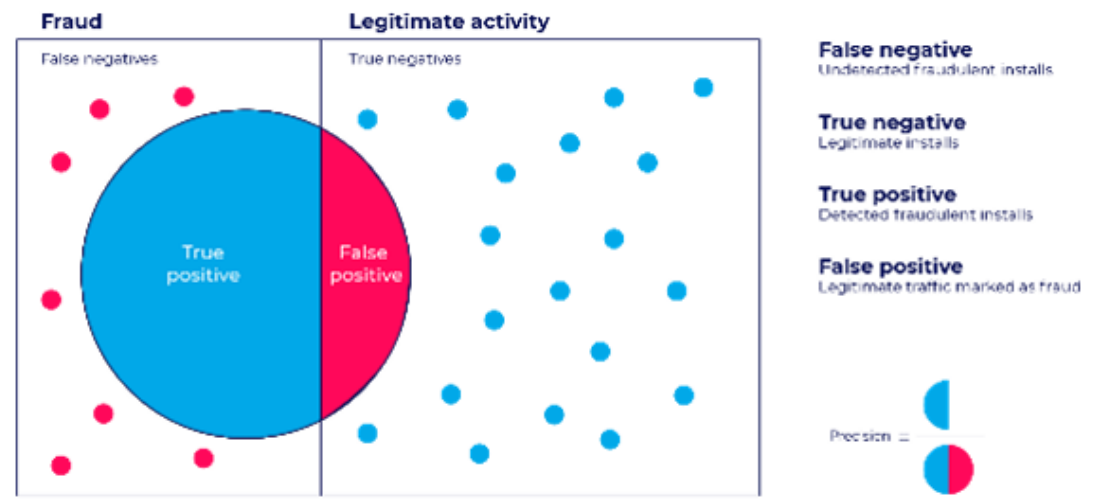

Figure: 2 False Positive

Source: appsflyer.com 
For instance, the statement and leave situations in for circles are supplanted as Task. Consequently, at that point, a tree design is produced from after-request tree crossing. After, a double-wise graphic design examination can be utilized to identify that kind of imitator. You have to design the ASTs to serve double imitator tests concurrently. These twin ASTs possess a natural graphical design with just three unique hubs in the main code test. In any case, further developed clone discovery strategies have been proposed, which can be summed up into twin strategies: chart coordinating based and amongst the double trees Deep neural networks (DNN)- based methodology. Shockingly, which has unavoidable downsides, in the first place, given two bits of code which vary in a couple of proclamations yet with the comparable dictating stream, upon its diagram coordinating enabled imitator location, they might be viewed as comparable, given that most of the code is indistinguishable. Then again, operational DNN-enabled imitator recognition is used to distinguish indistinguishable code imitators (for instance, using code closeness $S=$ 1.0). Therefore, if the uniformity edge is kept within the region of $S<1.0$, the yields would resemble the standard graphical-enabled/enabled- technique. Quite an obvious viewing that the chief code test possesses an additional limit call fprintf appearing differently in relation to the resulting code test. Upon a consideration to slacken up the code comparability limit, these double code tests are recognized, code imitators.

Forging forward with a dependence examination measure, changes $\{$ if, $j$, mdef $->n$ sseq, mdef $\mathrm{n}$ communicate condition are perceived as indicator-concerned components (that may disturb the worth of indicators) as a focal indicator within the chief model (second code model is operational using a comparable procedure). Regardless, fprintf can't impact any future profits of those elements. Appropriately, the bound security terms cannot be set in stone as these:

$\{\mathrm{i}<$ straightness $($ mdef $->$ sseq $)\} \wedge\{\mathrm{j}<$ straightness $($ mdef $->$ sseq) $\}$ \{coded $<$ long $($ gs $->$ codeword) $\} \wedge\{$ cid $<$ length (gs $->$ codeword) $\}$ independently. You would most apparently see that they are vague considering how the conditions fluctuate simply in factor names. Like this, they are substantial positives as they possess comparative pointer prosperity terms. Despite the way that an easygoing code closeness can recognize such imitators, it can similarly bring in a ton of bogus positives.

\section{Program SLicing}

The typical beginning stage is to name some specific kinds of factors as pointer-related factors initially. Those variables might possibly influence the platform, offset, or bound data of focusing on the indicator. For example, indicator augmentation and cluster list are the most well-known indicator-based factors. The major source base of this article uses reliance investigation to discover such indicator-based factors for every pointer on a capacity level granularity. Then, at that point, we send both forward and in reverse program cutting to choose related articulations containing pointer and pointer-related factors.

- Pointer Separating: The principal direct static code examination to gather every one of the pointers information from each capacity, including pointer affirmation type (for example, whole number or string, neighborhood factors, or worldwide factors). A pointer list is then created for each source program. Specifically, one can utilize an event splitter ANTLR and a stiff code examination device Joren to investigate encoding sentence structure. 


\section{Program Slicing Example}

int $i$;

int sum $=0$;

int product $=1$

for $(\mathrm{i}=0 ; \mathrm{i}<\mathrm{N} ; \mathrm{i++})$

\{

sum $=\operatorname{sum}+1$

product $=$ product ${ }^{*}$;

\}

write(sum):

write(product);

Original Program int $\mathrm{i}$;

int sum $=0$;

for $(\mathrm{i}=0 ; \mathrm{i}<\mathrm{N} ; \mathrm{i}++)$

\{

sum $=\operatorname{sum}+1$

\}

write(sum);

Slice on statement

"write(sum)"

Figure 3: Program slicing

Source: slideplayer.com

- Code Reliance Examination and Spoiling: A coordinated reliance diagram is made for every indicator pi inside the capacity where it is initially announced. The hubs of chart $\mathrm{N}$ address the identifiers in the capacity, and edges $\mathrm{E}$ address the reliance between hubs, which reflects exhibit ordering, tasks among identifiers, and boundaries of capacities. When the reliance chart is developed, we start with the objective pointer pi and navigate the reliance diagram to find all pointer-related factors in hierarchical and base-up bearings. This corrupting measure stops at work limits which further helps in maintaining optimum mathematical in the code detection strategies.

- Program Detachment: After one ought to have viably secured every one of the focal indicators and their contrasting indicator-based, whether forward and in invert structured slicing are passed on to disconnect code into indicator-lone code. Expecting an indicator-based dynamics overview, you ought to at first endeavor backward cutting: then carefully fabricate a retrogressive cut on each factor vi $\in \mathrm{V}$ around the completion of the limit and remove backward to perhaps add the declaration into the cut in the event that ensures data dependence as vi is there at the left-hand side of undertakings or limit of limits, which may possibly impact the worth of vi, in the cut. Illustratively, a trace of verbalization VI $=\mathrm{x}$ would be separated, anyway $\mathrm{y}=\mathrm{VI}$ can be disposed of since it can't disrupt the worth of vi. Anytime vi is all around (for instance, while/for circle), on the other hand, if-else/switch aspects. However, slicing is optimized to include those teleguided dependence announcements to the cut. This ensures a performing program cutting, and we can disengage one single limit into a couple of pointers confined limits. For instance, accepting which has ten indicators in a solitary limit should indicate separated limits drawn from this limit. One should not lose sight of the fact that it is feasible for one verbalization to incorporate different indicators. This kind of clarification will be picked in all of the intricate pointers. In expands, one in like manner needs to devise a guile technique to save the region of any picked clarifications in the principal source code for extra examination.

\section{Code Clone Detection Approach}

Twin-Finder+ maximizes a tree-based code imitation discovery strategy, viewed to have been naturally enunciated by Jiang et al., Which manufactures what is known as the 
Abstract Syntax Tree (AST) symbolism of the database program to unravel the code imitation by analyzing them side by side with charts in ASTs with a particular uniformity measuring unit. AST is ordinarily optimized through a tree symbolism enabled through compilers to bring out syntactic construction inherent in the code and to dissect the conditions among factors and explanations. The database upon which the code is sourced can be split by optimizing the dynamic code examination and eventually producing AST. At this juncture, one must embrace the thoughts of code similitude, highlight vectors, and similarly interconnected conceptions emanating through former dealings. You can presumably convey such a technique to identify code clones among disconnected pointer codes on the highest point of your space explicit cutting module.

After an occasion of packing a few parts of vectors, we use Location Special Hashing $(\mathrm{LSH})<25>$. Close neighbor addressing estimation reliant upon the Euclidean gap existing for the two vectors to bundle a vector pack, understanding fully well that LSH can hash twin equal vectors to a comparable hash worth and approaches addressing computation to shape gatherings. Envision that two-component vectors $\mathrm{Vi}$ and $\mathrm{Vj}$ tending to two code pieces, $\mathrm{Ci}$ and $\mathrm{Cj}$, independently. The code weight (without a doubt the quantity of AST center points) is implied as $\mathrm{S}(\mathrm{Ci})$ and $\mathrm{S}(\mathrm{Cj})$. The Euclidean gap $\mathrm{E}([\mathrm{Vi} ; \mathrm{Vj}])$ and hamming distance $\mathrm{H}([\mathrm{Vi} ; \mathrm{Vj}])$ among $\mathrm{Vi}$ and still up in the air as follows:

$\mathrm{E}([\mathrm{Vi} ; \mathrm{Vj}])=|| \mathrm{Vi}-\mathrm{Vj}|| 22(4) \mathrm{H}([\mathrm{Vi} ; \mathrm{Vj}])=|| \mathrm{Vi}-\mathrm{Vj}|| 1(5)$

The edge utilized for get-together can be approximated utilizing the Euclidean gap and hamming distance between two section vectors for twin ASTs T1 and T2 as such: E ([Vi; $\mathrm{Vj}]) \geq \mathrm{qH}([\mathrm{Vi} ; \mathrm{Vj}]) \approx \sqrt{\mathrm{L}}+\mathrm{R}(6)$

Putting in mind the explanation emanating off scenario 2, one can effectively conclude that $\sqrt{L}+R=p 2(1-S) \times(|\mathrm{T} 1|+|\mathrm{T} 2|)$, where $(|\mathrm{T} 1|+|\mathrm{T} 2|) \geq 2 \times \min (\mathrm{S}(\mathrm{Ci}), \mathrm{S}(\mathrm{Cj})$.

\section{- Bound Verification}

Presently, to officially check if the code clones distinguished by Twin-Finder+ are, in fact, code clones as far as indicator storage security, it becomes imperative to suggest an imitator authenticator network and optimize representative implementation as a check instrument. There are exists about three stages of imitator's confirmation:

1. Recursive inspecting code, imitators in clusters.

2. Send representative operations and requirements tackling for imitators check.

3. An input instrument to vector implanting in past code imitators' recognition module to work on the accuracy of bunching calculation and dispense with bogus positives.

\section{- Recursive Testing}

To work on including code clone tests in the groups, one can propose a recursive inspecting system to choose imitator tests for clone verification. Just know that it is feasible to separate one bunch into a few more modest bunches arbitrarily. Then, at that point, we select irregular code imitator tests from each more modest group place and bunch limit. After, it is alright to utilize representative execution in chose tests for additional clone checks. Note that the code clone tests are pointer detached code created from program cutting. Since representative operation needs the code culmination, one would then be able to plan the code imitators' tests to the first source code areas to perform halfway emblematic processes. 


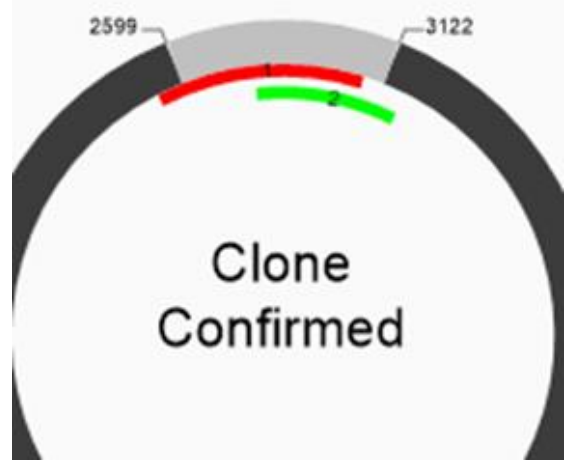

Figure 4: Code verification

Source: dnastar.com

\section{- Clone Verification}

Clustering calculation can't give any assurances as far as guaranteeing safe indicator access from all distinguished code clones. It is conceivable that two code parts are bunched together yet have diverse bound wellbeing conditions, particularly in the event that one utilizes a more modest code closeness. To additionally further develop the clone discovery precision of Twin-locater, we plan a clone confirmation technique to consider if the code imitator tests are even credible imitators. Allow $X=\{\mathrm{p} 1, \mathrm{p} 2, \mathrm{pn}\}$ become one of the confined strategies of indicator-enabled conditions as specialist factors, while representative carrying out the activity in every conceivable way, every way keeps a ton of essentials called the way conditions which should hold tight the execution of that way. Most importantly, we portray a nuclear condition, $A C()$, over $X$ is as $f(p 1, p 2$, pn), where $f$ is a cutoff that plays out the whole number procedure. Fundamentally, a situation over $\mathrm{X}$ can be a Boolean mix of ways analyzing the circumstances over X. Offer a clone pair evaluated from the past advance, and you can then perform delegate execution from starting to the farthest uttermost scopes of clone tests in surprising resource code dependent upon the spaces data (line measures of code).

The critical expert is utilized to explore the entirety of the potential ways existing in the code piece. We collect the entirety of as far as possible for each clone test after delegate execution is done. To manage perhaps lacking system state while performing insufficient significant execution, one can make the indicator-based segments in that code piece as specialist factors. You can gather the entirety of as far as possible for each clone test after specialist execution is done. Then, at that point, the assertion cycle is clear. It is moreover conceivable to send a pairwise evaluation of objectives between two clone tests. Two code clones are asserted as clear code clones if the obstructions are a wary match. Since we investigate pointers in our work, limitations don't cover the degree that the memory reference. Regardless, it is functional for delegate execution to make more than one heap of requirements since the clone tests might have diverse ways. For the current situation, we need to join together and coordinate the entirety of the limitations into one. There are some leaving instruments that can be utilized to settle and join such targets. Then, at that point, the attestation correspondence is prompt. A cutoff solver may even be utilized to verify the realness with the syntactic proportionality of clear conditions more than somewhere near one speculation. 


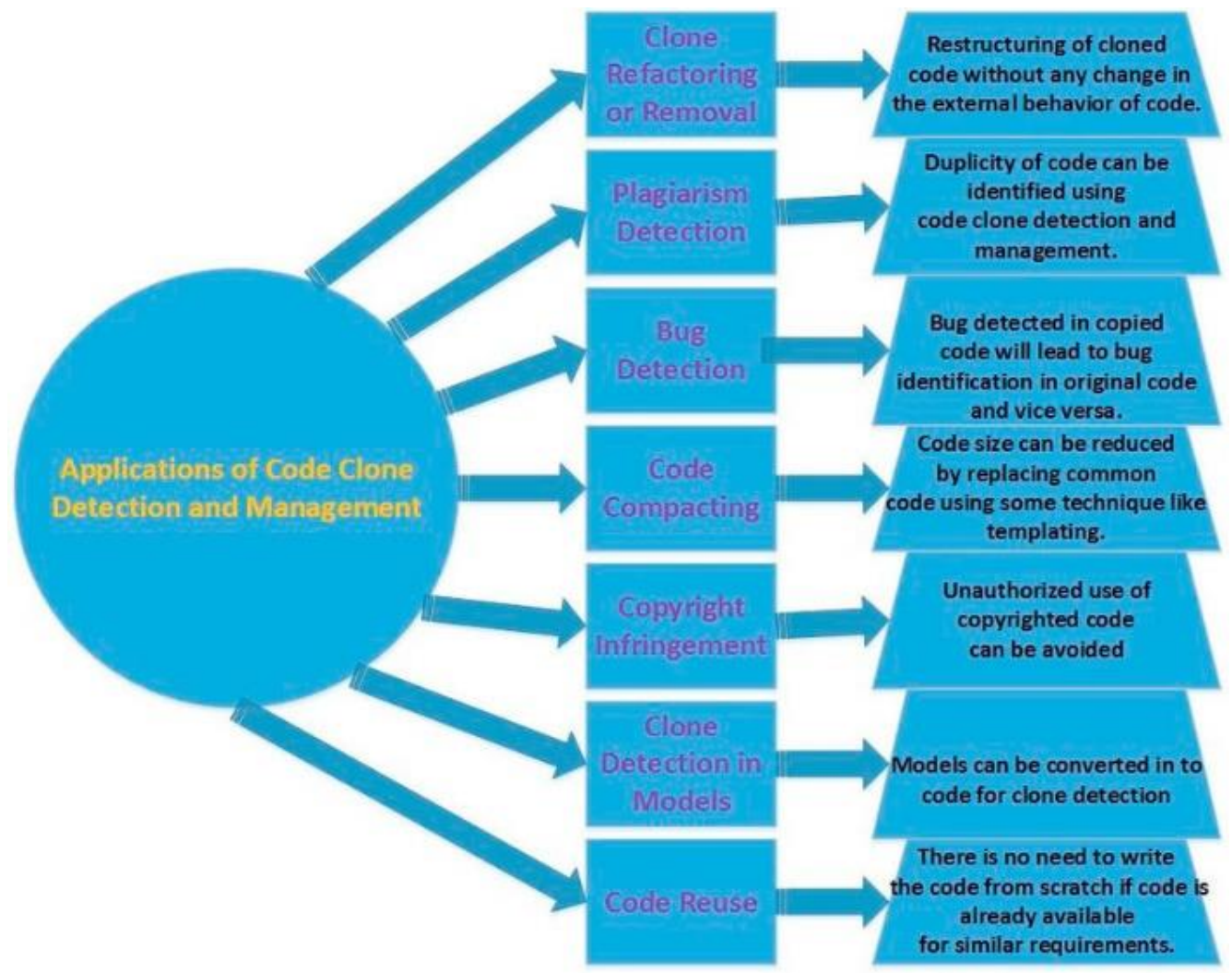

Figure 5: Code clone detection

Source: researchgate.net

\section{The guidelines of this ascertainment procedures are simplified thus:}

- Matching the Variables: when confirming if two arrangements of requirements are equivalent, we exclude the distinction of variable names. In any case, we need to coordinate with the factors between two requirements dependent on their reliance on target pointers. For example, two-pointer dereference $\mathrm{a}[\mathrm{i}]=0 \mathrm{~A} 0$ and $\mathrm{b}[\mathrm{j}]=0 \mathrm{~B} 0$, the ordering factors are I and j separately. During symbolic execution, the two of them will be supplanted as representative factors, and we couldn't care less about the names of the factors. Subsequently, we can infer reasoning which suggests I is comparable to $\mathrm{j}$ when the additional investigation is carried out. This earlier information can be effortlessly gotten through reliance investigation.

- Reduction: Given a memory wellbeing condition, it can possess an avalanche of straight disparities. For straightforwardness, the first step is to discover potentially less difficult articulation S 0, identical to S.

- Checking the Comparability: To exhibit two plans of prerequisites $\mathrm{S} 1==\mathrm{S} 2$, one would need to show the invalidation of $\mathrm{S} 1==\mathrm{S} 2$ is unclassifiable.

- Model: Note that, for instance, when we have two game plans of necessities, S1 = (y1 $\geq 10) \wedge(x 1 \geq 20)$ and $S 2=(y 2 \geq 10) \wedge(x 2 \geq 20)$, where $y 1$ is tantamount to $y 2$ and $x 1$ is indistinguishable from $\mathrm{x} 2$. 


\section{Formal Feedback to Vector Embedding}

A feedback system of interaction is an estimation, and it has been proposed that to diminish fake positives by tuning the part vectors burdens to the vector implanting's. The overall thought about our analysis is that we inspect the qualification existing amongst 2 ASTs by seeing some trees and find the differentiation in the center. You can then make additional numerical burdens to the component vectors of double code imitation to either augmentation or reducing the gap apparent among them subject to the yields from the imitation affirmation guide. When the weight is added, one can still execute the batching computation in code imitation acknowledgment style over a comparative code closeness edge game plan. Beware of this strategy as it can be carried out in various accentuations if we notice counterfeit positives arising through the clone inquiry pattern. In addition, you can anticipate that such bogus outcomes are discarded due to disgruntled vector space and out of bundle restrictions. Considering the code resemblance edge S, Usually, only two clone tests $(\mathrm{Ci}, \mathrm{Cj})$, contrasting AST sub-trees and incorporate vectors $(\mathrm{Vi}, \mathrm{Vj})$ pointing to 2 code imitations as resultant effects of data, and which uses an associate work LCS to find the Longest Typical Delayed consequence amongst both programs of sub-trees. Exactly as soon as the code imitation tests are graphically carried out, which means one should commence by investigating if the necessities, gotten from past legitimate affirmation guides, are one. Then the info procedure after is driven as two folds: (1) If they truly share comparable limits, we take out the unique trees of lower magnitude, which presently acknowledges they will not impact the yield of necessities. This cycle is to guarantees the extra trees are indistinct, so they will be recognized as code clones later on, which may be proposed to authenticate a situation whereby these twin code tests will not be seen as code imitators later on. Finally, the info can run in a circle style to clear out fake positives (Ujwala et al., 2012). The eventual outcome for our feedback circle would be that no more counterfeit positives would be taken note of.

Model: Assuming the segment vectors are $<7,2,2,2,0,1,1,1,1,1>$ independently, where the organized components of vectors are occasion counts of the appropriate center points: ID, Consistent, ArrayRef, Assignment, and For Considering the edge portrayed in condition. These two code pieces will be gathered as clones. We first recognize these two exceptional center points of every tree during the analysis circle by obtaining the LCS. Expecting we basically the weight $\delta=2$ and add it to the contrasting estimation in the segment vectors, you can secure the invigorated component vectors as $<1>$. You can register the Euclidean distance of these two revived segment vectors once more, and they will be starting now, not satisfied inside the edge pi. Consequently, we can take out such sham empowering focuses later on.

It is moreover worth zeroing in on that these forms of our analysis estimation helped a not too open circle learning-put together movement to work with respect to the versatility of our indicator-based code imitators' area structure. Since this system gives advantages by official measurements and can bring down some bogus positives without individual undertakings included, it has to be significantly leveraged to aid in informatics analysis using code-based detection mechanisms.

\section{CONCLUSION}

It has been established through the wordings of this article on the topical issue of integrated engines for code detection that there are very authentic approaches that must be religiously followed to detect the details of the various codes properly. When cloning 
systems are involved, it takes a heightened level of analytics to discover such information. No doubt that even internal concepts like false positives, program slicing, recursive samplings all point out that there is no very water-tight approach towards detecting code to further better analysis using formal feedback to vector embedding. Be that as it may, it is trusted that this article would have proven useful for statistical firms and organizations and those in the communications and intelligence unit of military formations who would naturally need all these deep-seated approaches to unravel hideous information about a certain phenomenon.

\section{REFERENCES}

B. S. Bread cook, Defined duplication in strings: Calculations and an application to programming upkeep, SIAM Diary on Processing 26 (5) (1997) 1343-1362.

D. Baxter, C. Pidgeon, M. Mehlich, Dms/SPL reg: program transformations for pragmatic, adaptable programming development, in Computer programming, 2004. ICSE 2004. Procedures. 26th Global Meeting on, IEEE, 2004, pp. 625-634.

H. Xue, S. Sun, G. Venkataramani, T. Lan, AI-based investigation of program parallels: An exhaustive report, IEEE Access 7 (2019) 65889-65912.

L. Jiang, G. Misherghi, Z. Su, S. Glondu, Deckard: Versatile and precise tree-based discovery of code clones, in Procedures of the 29th international gathering on Programming, IEEE PC Society, 2007, pp. 96-105.

M. Gabel, Z. Su, An investigation of the uniqueness of source code, in Procedures of the eighteenth ACM SIGSOFT global conference on Establishments of computer programming, ACM, 2010, pp. 147-156.

M. Kim, V. Sazawal, D. Notkin, G. Murphy, An experimental investigation of code clone lineages, in ACM SIGSOFT Programming Notes, Vol. 30, ACM, 2005, pp. 187-196.

Movva, L., Kurra, C., Koteswara Rao, G., Battula, R. B., Sridhar, M., \& Harish, P. (2012). Underwater Acoustic Sensor Networks: A Survey on MAC and Routing Protocols. International Journal of Computer Technology and Applications, 3(3).

Narayana, S. L., Suneetha Devi J., Bhargav Reddy I., Harish Paruchuri. (2012). Optimizing Voice Recognition using Various Techniques. CiiT International Journal of Digital Signal Processing, 4(4), 135-141

T. Kamiya, S. Kusumoto, K. Inoue, Ccfinder: a multilinguistic token-based code clone discovery framework for enormous scope source code, IEEE Exchanges on Programming 28 (7) (2002) 654-670.

Ujwala, D., Ram Kiran, D. S., Jyothi, B., Fathima, S. S., Paruchuri, H., Koushik, Y. M. S. R. (2012). A Parametric Study on Impedance Matching of A CPW Fed T-shaped UWB Antenna. International Journal of Soft Computing and Engineering, 2(2), 433-436.

Z. Li, S. Lu, S. Myanmar, Y. Zhou, Cp-digger: Discovering duplicate glue and related bugs in huge scope programming code, IEEE Exchanges on computer programming 32 (3) (2006) 176-192. 